\title{
Felted Paper Circuits Using Joomchi
}

Nicholas A. Knouf

Cinema and Media Studies

Program

Wellesley College

Wellesley, MA, 02481, USA

nknouf@wellesley.edu

\begin{abstract}
The integration of electronics and paper is a burgeoning topic for tangible interaction. Here we show how to use the Korean technique of hand-felting paper known as joomchi to embed electronics in paper after the sheet forming process. This method requires the use of specialized papers that are amenable to joomchi. We present embedded LEDs, multilayer circuit "sheets", and speakers. While labor-intensive, joomchi both enables one to completely integrate paper and electronics, while also making tangible connections between ancient and modern craft.
\end{abstract}

\section{Author Keywords}

paper circuits; hanji; joomchi; craft

\section{ACM Classification Keywords}

J.5 [Arts and Humanities]: Arts, fine and performing

\section{Introduction}

The deep integration of paper and electronics is a growing area of interest for human-computer interaction researchers [7]. Given thousands of years of experience with different forms of paper around the world, paper provides a versatile material for new forms of tangible interaction due to its affordances of ubiquity, familiarity, and ease of modification. 
Recent work surrounding the integration of paper and electronics has followed a number of different trajectories. Researchers have shown how to embed electronic components during the forming of individual sheets of paper, developing what they call "pulp-based computing" [3]. The combination of thermochromic inks and layers of paper have enabled artists and designers to create works that change color in response to touch and heat $[6,23,24]$. New developments in inexpensive conductive ink and paint have allowed for the printing and/or drawing of circuits on the surface of paper [9, 20, 21]. Shape-memory alloys have provided for novel movement mechanisms [14, 22, 19]. Copper tape has enabled the construction of "circuit stickers" that can be used to teach the basics of electronics $[15,16]$. Various forms of cutting and depositing copper traces on the surface of paper have allowed for new kinds of flexible speakers $[10,18,17]$. Combinations of some or all of these techniques have suggested new ways to think about the intersection of electronics and books [4, 13, 12]. In sum, there is now a vibrant community of researchers tracing out various potentialities for paper computing.

We contribute to this body of work by showing how to use the Korean method of hand-felting paper called joomchi to embed electronic components into the paper after the sheets have been formed. Joomchi requires specialized papers with particular material qualities such as the Korean paper called hanji, a long-fibered paper made from parts of the paper mulberry tree [8]. Hanji has recently become more well-known in the West through the dedicated advocacy of the artist and researcher Aimee Lee. Joomchi enables one to take a stack of multiple sheets of paper and, through repeated hand agitation with water, felt the individual sheets into a single sheet, with the electronic components embedded within the paper itself. This creates a seamless integration of paper and electronics without the complication and difficulty of processing fibers and forming paper by hand.

\section{Paper Characteristics}

While there is a large amount of research into the integration of paper and electronics, few of these projects have addressed the specific materiality of particular kinds of paper, although the pulp-based computing research of Coelho et al. is a notable exception [3]. We think this is a significant oversight, as different types of paper have unique characteristics depending on the choice of fiber, method of separating fibers, and sheet-forming process, amongst other considerations. Here we give a brief overview of why we are working with hanji and why such paper is necessary for joomchi, illustrating how long-fibered papers like hanji could be of interest to the TEI community.

Papermaking is an ancient craft, and all manner of plant fibers have been used to make paper, such as cotton, wood pulp, or the leaves of invasive weeds [5]. Here we are interested in the particular qualities of the inner bark or "bast" of paper mulberry trees, known as dak in Korea or kozo in Japan (hanji is almost exclusively made of dak, while Japanese paper, known as washi, can be made from kozo or other plant fibers) $[1,8]$. Dak fibers are rather long in comparison to other plant fibers (on the order of $12 \mathrm{~mm}$, in comparison with $4 \mathrm{~mm}$ for Western-style pulp), and the process of hand-beating dak in order to make pulp does not chop up these fibers but allows them to retain their length $[1,8]$. The particularities of the hanji sheet-forming process also allows for an incredibly strong sheet, so much so that hanji has been used for windows, floors, shoes, teapots, umbrellas, and armor, amongst many other objects [8].

No matter the source fiber, "pulp" generally refers to these processed plant fibers suspended in water. This pulp be- 
comes paper through hydrogen bonding of cellulose between the fibers themselves as the water evaporates from the pulp after a sheet has been formed [1, pg. 290]. Hanji's strength comes primarily from this hydrogen bonding of its long fibers. Because of this strength, the fibers between two or more sheets of hanji can be "felted" together through joomchi, a craft that has existed for hundreds of years and has recently become well-known amongst papermakers due to the teaching of workshops by artists such as Jiyoung Chung and Aimee Lee [2, 8]. Joomchi involves wetting sheets of hanji (or washi made of kozo) and, through various forms of hand agitation and manipulation, melding multiple sheets into one through the formation of new hydrogen bonds between fibers of the original sheets.

Because of the merging of multiple layers of hanji into a single sheet through joomchi, any inclusions (for example, electronic components) that we place on interior sheets become a part of the new single sheet of hanji. This allows us to take advantage of the characteristics of hanji without having to embed the electronics as inclusions at the time of sheet formation, an option unavailable to most given the specialized equipment and training necessary to make hanji or other types of specialized paper. We are also able to draw upon the wide variety of dyed papers available on the market. While labor intensive, joomchi therefore enables anyone to embed electronics into paper (rather than placing them on the surface, as in many other paper electronics projects), while at the same time extending an ancient craft into new directions.

\section{Example Joomchi Procedure}

We use a slightly modified form of joomchi from that described by Jiyoung Chung in her book Joomchi \& Beyond

[2]. Credit goes to Chung for creating easy-to-follow recipes for different types of joomchi; we nevertheless outline the most basic steps here, as well as provide visual documentation in Figure 1. Joomchi has many variations that could be of use to the paper computing designer, and we encourage those interested to examine Chung's book.

1. On a single sheet of hanji (Japanese washi made from kozo can also be used), sew the electrical circuit desired (Figure 1a).

2. Place additional sheets of hanji above and below the sewn sheet(s) from Step 1 (Figure 1b).

3. Thoroughly wet with a spray bottle all sheets of hanji. It is helpful to wet each sheet individually, building up the stack sheet by sheet, and ensuring that there are few to no air bubbles between the sheets (Figure 1c).

4. For the first "round" of joomchi, accordion fold along the horizontal edge, then roll into a cylinder. Agitate the cylinder by compressing it evenly in your hand, ensuring that all parts of the cylinder receive equal agitation. The cylinder needs to remain wet throughout this process, so spray with additional water as needed. Agitate for ten minutes (Figures 1d and 1e).

5. Unroll the cylinder, and roll it up in the opposite direction. Agitate for 10 minutes.

6. Unroll and unfold the sheet, then accordion fold in the vertical direction. Repeat steps 4 and 5 .

Unroll and unfold the sheet. At this point you will have agitated for around 40 minutes (Figure 1f). The next step can be repeated ad infinitum, but should be done at least four to five times. 


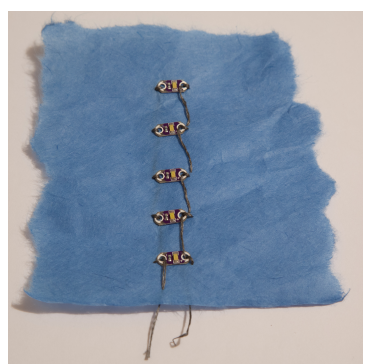

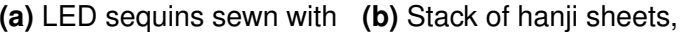
conductive thread

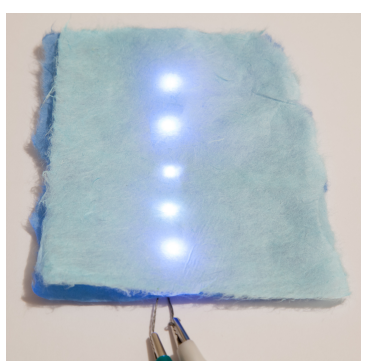
before wetting: light blue bottom layer, dark blue

sewn layer, light blue top layer

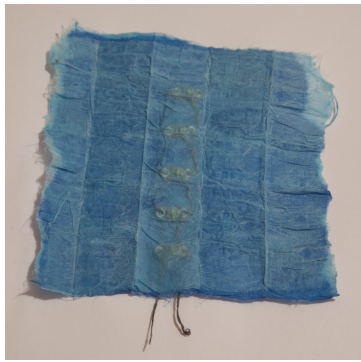

(f) Unrolled and unfolded hanji stack after four 10 minute "rounds" of joomchi

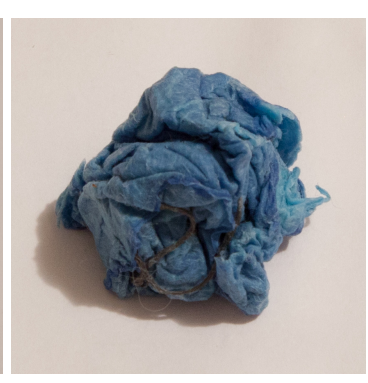

(g) Crumpled and wetted hanji ball

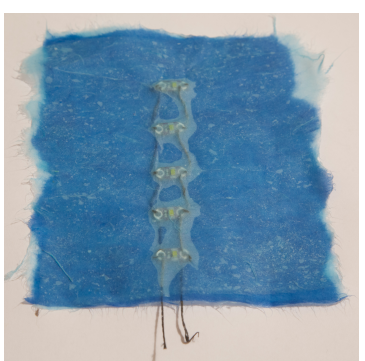

(c) Thoroughly wetted hanji (d) Accordion folded hanji stack

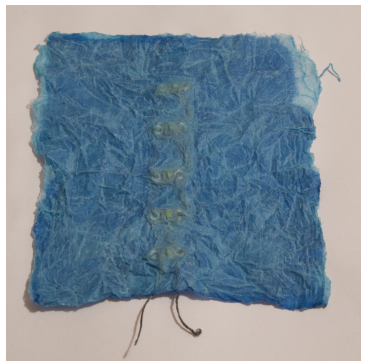

(h) Uncrumpled hanji ball

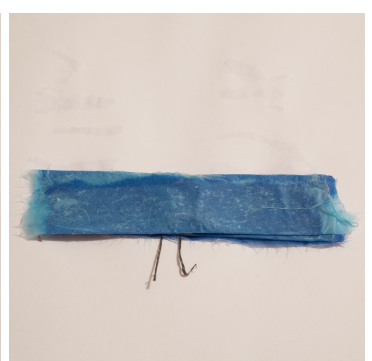

stack

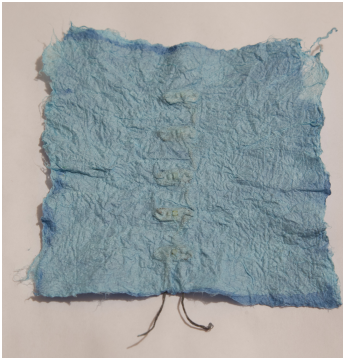

(i) Dried sheet of hanii after (i) a total of around 110 minutes of joomchi

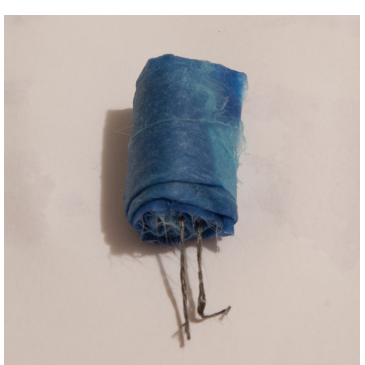

(e) Accordion folded stack rolled into a cylinder

Figure 1: Illustration of joomchi steps 


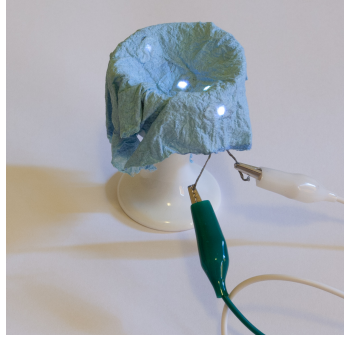

(a) Hanji sheet from Figure 1 that has been re-wetted, draped over an how the underlying object, showing

Figure 2: Draping circuits made of hanji after joomchi.

7. Re-wet the sheet and crumple it into a ball. Agitate by squeezing the ball evenly for 10 minutes (Figures $1 \mathrm{~g}$ and $1 \mathrm{~h})$.

A properly felted sheet of hanji will take at least 80 minutes of hand agitation, and likely upwards of 120 minutes (Figures $1 \mathrm{i}$ and $1 \mathrm{j}$ ). The amount of agitation needed is dependent on the original size of the sheet (in this example the sheet was around 5" on a side) as well as the number of sheets in the stack ${ }^{1}$. As is clear from the figures and the list of steps, joomchi is likely not amenable to the embedding of large, inflexible components, although new techniques might make this possible. For now, inclusions need to be small and/or malleable, such as small LED sequins and conductive thread. As well, inclusions need to be able to withstand being soaked in water, at least for the duration of the joomchi and drying processes. So far standard LED

${ }^{1}$ There is often some shrinkage in both horizontal and vertical dimensions after joomchi. sequins have continued to work even after being continually soaked in water during joomchi, but their long-term viability is an open question. Conductive threads will likely move around somewhat during joomchi, thus threads carrying differing signals need to be separated by a reasonable distance at the time of sewing.

Following joomchi, the sheet is by default relatively stiff, with the feeling of leathery skin. This can be modified by gently rubbing the sheet in one's hands to soften the paper [2]. With enough time, the paper takes on the characteristics of cloth, with all of the draping possibilities that implies.

\section{Further examples}

The example shown in Figure 1 involved sewing five LilyPad LED sequins $^{2}$ to a sheet of hanji using conductive thread. We show here other examples of felted paper electronics made through joomchi.

- It is possible to take a sheet of hanji after joomchi and, by re-wetting it, allow it to drape and take the form of an underlying object. We took the sheet shown in Figure 1 and draped it over an egg cup and let it dry. The resulting sheet retains its shape even without the underlying object (see Figure 2).

- Because of hanji's strength, we can have electronic inclusions that can be seen from the front and back of the sheet, creating what we could call a "sheet circuit". By using layers of hanji as insulators between two individual layers that have electronic inclusions, we can replicate the insulating material of printed circuit boards (see Figure 3).

\footnotetext{
${ }^{2}$ https://www.sparkfun.com/products/10081
} 


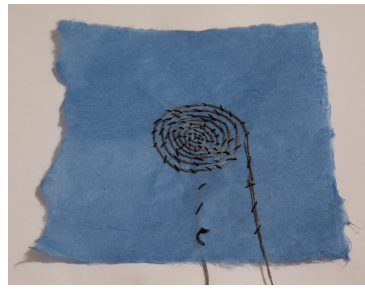

(a) Embroidered speaker made from conductive thread, front.

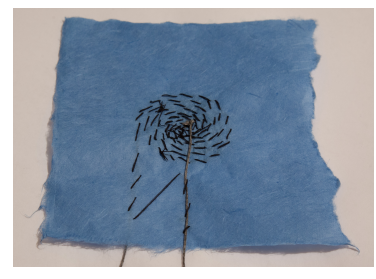

(b) Embroidered speaker made from conductive thread, back.

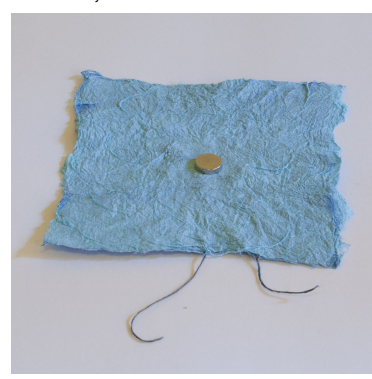

(c) Embroidered speaker after joomchi, showing placement of neodymium magnet.

Figure 4: Creating a paper speaker through joomchi.

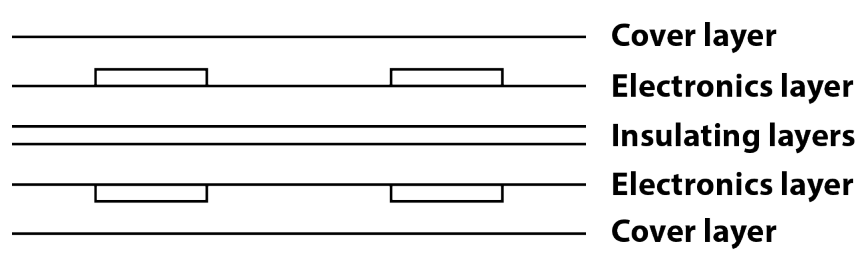

(a) Diagram of a hanji stack forming a "sheet circuit", shown end-on before the individual sheets have been felted together through joomchi.

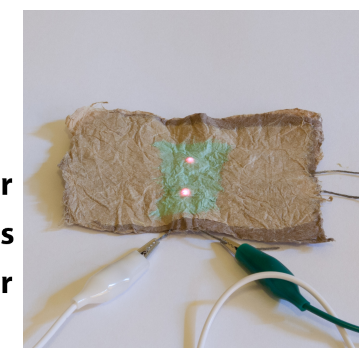

(b) Front side of a hanji "sheet circuit" after joomchi.

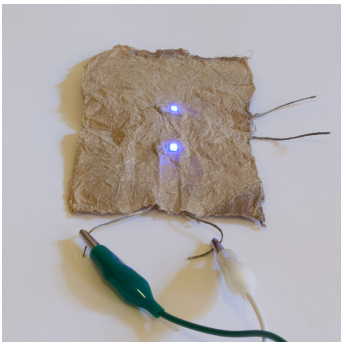

(c) Back side of a hanji "sheet circuit" after joomchi.

Figure 3: Creating a "sheet circuit".

- We can also create paper speakers by sewing conductive thread in a spiral pattern and attaching a powerful neodymium magnet, drawing upon the work of Hannah Perner-Wilson (see Figure 4) [10, 11].

These examples act as proofs of concept that show some of the potential for the embedding of electronics in paper through joomchi.

\section{Future Work}

The examples of paper circuits in this paper are the beginning of our research into the intersection of hanji, electronics, and joomchi. We intend to further refine our procedure to make the sewing more resilient to joomchi, as well as considering other methods of joomchi besides the basic one used in this paper. We will also explore the possibility of using conductive inks or paints to create bend and capacitive sensors, as well as the embedding of NFC tags for the storage of small amounts of data within the paper itself. The purpose of our investigations is to weave together the most appropriate affordances of paper and electronics. In sum, we hope that others consider how the specific material qualities of different types of paper could be useful in creating novel paper circuits, while at the same time enabling one to extend old forms of craft into new directions.

\section{Acknowledgements}

This project was funded in part through a Wellesley College faculty award. Thanks to the two anonymous reviewers for their comments. Many thanks to Lisa Cirando for teaching me all I know about papermaking, hanji, and joomchi. I would also like to thank Jeff Stout for insightful conversations about paper circuits.

\section{REFERENCES}

1. Timothy Barrett. 2005 [1983]. Japanese Papermaking: Traditions, Tools, and Techniques. Floating World Editions, Easthampton, Massachussetts.

2. Jiyoung Chung. 2011. Joomchi \& Beyond. Beyond \& Above, Providence.

3. Marcelo Coelho, Lyndl Hall, Joanna Berzowska, and Pattie Maes. 2009. Pulp-based Computing: A 
Framework for Building Computers out of Paper. In $\mathrm{CHI}$ '09 Extended Abstracts on Human Factors in Computing Systems (CHI EA '09). ACM, New York, NY, USA, 3527-3528. DOI :

http://dx.doi.org/10.1145/1520340.1520525

4. Natalie Freed, Jie Qi, Cristina Sylla, and Pedro Branco. 2011. Beyond the Binding: Exploring the Future Book. In Proceedings of the 8th ACM Conference on Creativity and Cognition (C\&C '11). ACM, New York, NY, USA, 445-446. DOI : http://dx.doi.org/10.1145/2069618.2069742

5. Dard Hunter. 1978 [1947]. Papermaking: The History and Technique of an Ancient Craft. Dover Publications, New York.

6. Tatsuya Kaihou and Akira Wakita. 2013. Electronic Origami with the Color-changing Function. In Proceedings of the Second International Workshop on Smart Material Interfaces: Another Step to a Material Future (SMI '13). ACM, New York, NY, USA, 7-12. DOI : http://dx.doi.org/10.1145/2534688.2534690

7. Fredéric Kaplan and Patrick Jermann. 2010. PaperComp 2010: First International Workshop on Paper Computing. In Proceedings of the 12th ACM International Conference Adjunct Papers on Ubiquitous Computing - Adjunct (UbiComp '10 Adjunct). ACM, New York, NY, USA, 507-510. DOI :

http://dx.doi.org/10.1145/1864431.1864500

8. Aimee Lee. 2012. Hanji Unfurled: One Journey into Korean Papermaking. The Legacy Press, Ann Arbor.

9. David A. Mellis, Sam Jacoby, Leah Buechley, Hannah Perner-Wilson, and Jie Qi. 2013. Microcontrollers As Material: Crafting Circuits with Paper, Conductive Ink, Electronic Components, and an "Untoolkit". In
Proceedings of the 7th International Conference on Tangible, Embedded and Embodied Interaction (TEI '13). ACM, New York, NY, USA, 83-90. DOI : http://dx.doi.org/10.1145/2460625.2460638

10. Hannah Perner-Wilson. 2011. A Kit-of-No-Parts. Master's thesis. Massachusetts Institute of Technology.

11. Plusea. 2012. Embroidered Fabric Speaker. (2012). Retrieved January 5, 2017 from http://www.instructables.com/id/ Embroidered-Fabric-Speaker/.

12. Jie Qi. 2012. The Fine Art of Electronics: Paper-Based Circuits for Creative Expression. Master's thesis. Massachusetts Institute of Technology.

13. Jie Qi and Leah Buechley. 2010. Electronic Popables: Exploring Paper-based Computing Through an Interactive Pop-up Book. In Proceedings of the Fourth International Conference on Tangible, Embedded, and Embodied Interaction (TEI '10). ACM, New York, NY, USA, 121-128. DOI :

http://dx.doi.org/10.1145/1709886.1709909

14. Jie Qi and Leah Buechley. 2012. Animating Paper Using Shape Memory Alloys. In Proceedings of the SIGCHI Conference on Human Factors in Computing Systems (CHI '12). ACM, New York, NY, USA, 749-752. DOI :

http://dx.doi.org/10.1145/2207676.2207783

15. Jie Qi and Leah Buechley. 2014. Sketching in Circuits: Designing and Building Electronics on Paper. In Proceedings of the SIGCHI Conference on Human Factors in Computing Systems (CHI '14). ACM, New York, NY, USA, 1713-1722. DOI :

http://dx.doi.org/10.1145/2556288.2557391 
16. Jie Qi, Andrew "bunnie" Huang, and Joseph Paradiso. 2015. Crafting Technology with Circuit Stickers. In Proceedings of the 14th International Conference on Interaction Design and Children (IDC '15). ACM, New York, NY, USA, 438-441. DOI :

http://dx.doi.org/10.1145/2771839.2771873

17. Jess Rowland. 2013. Flexible Audio Speakers for Composition and Art Practice. Leonardo Music Journal 23 (2013), 33-36.

18. Jess Rowland and Adrian Freed. 2012. Flexible Surfaces for Interactive Audio. In Proceedings of the 2012 ACM International Conference on Interactive Tabletops and Surfaces (ITS '12). ACM, New York, NY, USA, 315-318. DOI :

http://dx.doi.org/10.1145/2396636.2396688

19. Greg Saul, Cheng Xu, and Mark D. Gross. 2010. Interactive Paper Devices: End-user Design \& Fabrication. In Proceedings of the Fourth International Conference on Tangible, Embedded, and Embodied Interaction (TEI '10). ACM, New York, NY, USA, 205-212. DOI :

http://dx.doi.org/10.1145/1709886.1709924

20. Michael Shorter, Jon Rogers, and John McGhee. 2014a. Enhancing Everyday Paper Interactions with Paper Circuits. In Proceedings of the 2014 Conference on Designing Interactive Systems (DIS '14). ACM, New York, NY, USA, 39-42. DOI :

http://dx.doi.org/10.1145/2598510.2598584
21. Michael Shorter, Jon Rogers, and John McGhee. 2014b. Practical Notes on Paper Circuits. In Proceedings of the 2014 Conference on Designing Interactive Systems (DIS '14). ACM, New York, NY, USA, 483-492. DOI : http://dx.doi.org/10.1145/2598510.2602965

22. Paul Strohmeier, Kaja Vembe Swensen, Cameron Lapp, Audrey Girouard, and Roel Vertegaal. 2012. A Flock of Birds: Bringing Paper to Life. In Proceedings of the Sixth International Conference on Tangible, Embedded and Embodied Interaction (TEI '12). ACM, New York, NY, USA, 333-334. DOI : http://dx.doi.org/10.1145/2148131.2148208

23. Kohei Tsuji and Akira Wakita. 2013. Transience: Aesthetics of Dynamic Colors Inside Japanese Calligraphy. In Proceedings of the 7th International Conference on Tangible, Embedded and Embodied Interaction (TEI '13). ACM, New York, NY, USA, 387-388. DOI :

http://dx.doi.org/10.1145/2460625.2460706

24. Takahiro Tsujii, Naoya Koizumi, and Takeshi Naemura. 2014. Inkantatory Paper: Dynamically Color-changing Prints with Multiple Functional Inks. In Proceedings of the Adjunct Publication of the 27th Annual ACM Symposium on User Interface Software and Technology (UIST'14 Adjunct). ACM, New York, NY, USA, 39-40. DOI :

http://dx.doi.org/10.1145/2658779.2659103 